\title{
Non-equilibrium processes in $p+$ Ag collisions at GeV energies
}

\author{
M. Fidelus, ${ }^{1}$ D. Filges,${ }^{2,3}$ F. Goldenbaum, ${ }^{2,3}$ L. Jarczyk, ${ }^{1}$ B. Kamys, ${ }^{1,}{ }^{*}$ M. Kistryn, ${ }^{4}$ St. Kistryn, ${ }^{1}$ E. Kozik, ${ }^{4}$ \\ P. Kulessa, ${ }^{4}$ H. Machner, ${ }^{5}$ A. Magiera, ${ }^{1}$ B. Piskor-Ignatowicz, ${ }^{1,2,3}$ K. Pysz, ${ }^{4}$ Z. Rudy, ${ }^{1}$ \\ Sushil K. Sharma, ${ }^{1,2,3}$ R. Siudak, ${ }^{4}$ and M. Wojciechowski ${ }^{1}$ \\ (PISA Collaboration) \\ ${ }^{1}$ M. Smoluchowski Institute of Physics, Jagiellonian University, Lojasiewicza 11, 30348 Kraków, Poland \\ ${ }^{2}$ Jülich Center for Hadron Physics, Forschungszentrum Jülich, 52425 Jülich, Germany \\ ${ }^{3}$ Institut für Kernphysik, Forschungszentrum Jülich, 52425 Jülich, Germany \\ ${ }^{4}$ H. Niewodniczański Institute of Nuclear Physics PAN, Radzikowskiego 152, 31342 Kraków, Poland \\ ${ }^{5}$ Universität Duisburg-Essen, Fakultät für Physik, Lotharstrasse 1, 47048 Duisburg, Germany
}

(Received 18 August 2017; published 27 December 2017)

\begin{abstract}
The double differential spectra $d^{2} \sigma / d \Omega d E$ of $p, d, t,{ }^{3,4,6} \mathrm{He},{ }^{6,7,8,9} \mathrm{Li},{ }^{7,9,10} \mathrm{Be}$, and ${ }^{10,11,12} \mathrm{~B}$ were measured at seven scattering angles, $15.6^{\circ}, 20^{\circ}, 35^{\circ}, 50^{\circ}, 65^{\circ}, 80^{\circ}$, and $100^{\circ}$, in the laboratory system for proton induced reactions on a silver target. Measurements were done for three proton energies: 1.2, 1.9, and 2.5 GeV. The experimental data were compared to calculations performed by means of two-step theoretical microscopic models. The first step of the reaction was described by the intranuclear cascade model INCL4.6 and the second one by four different models (ABLA07, GEM2, GEMINI++, and SMM) using their standard parameter settings. Systematic deviations of the data from predictions of the models were observed. The deviations were especially large for the forward scattering angles and for the kinetic energy of emitted particles in the range from about 50 to $150 \mathrm{MeV}$. This suggests that some important non-equilibrium mechanism is lacking in the present day microscopic models of proton-nucleus collisions in the studied beam energy range.
\end{abstract}

DOI: 10.1103/PhysRevC.96.064618

\section{INTRODUCTION}

A dominating role of the two-step mechanism of spallation reactions induced by protons on the atomic nuclei is usually accepted. The reaction process is modeled as an intranuclear cascade of nucleon-nucleon and nucleon-pion collisions leading to equilibration of the remnant nucleus which then deexcites by evaporation, fragmentation, and/or fission. Such an equilibrium emission of particles should result in the presence of quasi-isotropic angular distributions of low-energy light charged particles (LCPs), i.e., $\mathrm{H}$ and $\mathrm{He}$ isotopes not heavier than ${ }^{4} \mathrm{He}$, as well as intermediate mass fragments (IMFs), i.e., particles lighter than fission products but heavier than LCPs. However, high-energy products of the intranuclear cascade, i.e., nucleons and pions, should manifest their presence by non-isotropic (forward peaked) angular distributions and high-energy tails of their energy spectra. It is, however, frequently observed that angular distributions of composite LCPs as well as those of IMFs are forward peaked and the corresponding energy spectra extend to larger energies than predicted by emission of particles from the equilibrated residual nuclei [1-15]. Such an effect was reported both at proton energies of several $\mathrm{GeV}(4.9-5.5 \mathrm{GeV})[12,14,15]$ and at low energies $(0.18-0.48 \mathrm{GeV})[3,6,10]$ for light (e.g., nickel [3]) as well as for heavy (e.g., uranium [15]) nuclei. This indicates that the presence of the non-isotropic, high-energy contribution to the spallation spectra is a general property. It was shown [16] that this contribution can be modeled for

*Corresponding author: ufkamys@cyf-kr.edu.pl emitted LCPs by surface coalescence of nucleons originating from the first stage of the collision process, i.e., from the intranuclear cascade. Significant improvement of the data description was reported both for $2.5 \mathrm{GeV}$ protons bombarding an $\mathrm{Au}$ target and for $0.542 \mathrm{GeV}$ neutrons impinging onto $\mathrm{Cu}$ and $\mathrm{Bi}$ targets due to the application of the Liége intranuclear cascade model INCL4.3 [16], which implements the above mechanism. The same physical picture was extended with success also to light IMFs $(\mathrm{A}<9)$ in the new version (INCL4.6) of this model [17]. However, it was recently shown for $0.48 \mathrm{GeV}$ proton induced spallation reactions on a silver target [18] that, while the model ensures the qualitative agreement with experimental IMF data, the high-energy tails of the spectra are strongly overestimated. It was also observed [19] that the production cross sections of isotopically identified residual nuclei with $Z=41-45$ from reactions induced by ${ }^{136} \mathrm{Xe}$ projectiles at $0.5 \mathrm{GeV} /$ nucleon on a hydrogen target are systematically underestimated by the model. Results of these two investigations might suggest that the applied microscopic model is not able to reproduce well the spallation cross sections for intermediate mass target nuclei at proton beam energies of around $0.5 \mathrm{GeV}$. It is important to know whether the observed disagreement vanishes at higher energies. Present investigations were undertaken to study experimentally the emission of LCPs and light IMFs from spallation reactions induced by protons of intermediate energies (1.2, 1.9, and $2.5 \mathrm{GeV}$ ) on silver nuclei. The experimental procedure and results are presented in the second section of the paper. The obtained data are compared with predictions of a two-step microscopic model in the third, section whereas the summary of results and the conclusions appear in the last section. 


\section{EXPERIMENTAL RESULTS}

The experiment was realized using the internal beam of the cooler synchrotron (COSY) of the Research Center in Jülich. The apparatus and experimental procedure were identical with those described in detail in the recent publication concerning spallation reactions induced by protons on an aluminum target [5]. Double differential cross sections $d^{2} \sigma / d \Omega d E$ were measured at seven scattering angles $15.6^{\circ}, 20^{\circ}, 35^{\circ}, 50^{\circ}, 65^{\circ}$, $80^{\circ}$, and $100^{\circ}$ for isotopically identified $\mathrm{H}, \mathrm{He}, \mathrm{Li}, \mathrm{Be}$, and $\mathrm{B}$ as well for elementally identified $\mathrm{C}, \mathrm{N}$, and $\mathrm{O}$ ejectiles.

A self-supporting silver target of $580 \mu \mathrm{g} / \mathrm{cm}^{2}$ thickness was used. It was turned by $65^{\circ}$ with respect to the beam direction to ensure approximately the same effective thickness for products of the reactions flying in the direction of all detectors. The target was bombarded by the internal proton beam of COSY which operated in the so-called supercycle mode. This consists in alternating several cycles of the accelerator for each beam energy $(1.2,1.9$, and $2.5 \mathrm{GeV})$ to guarantee the same experimental conditions for all energies. Each cycle consisted of proton injection from the JULIC cyclotron, acceleration of protons with the beam circulating in the COSY ring below the target, and irradiation of the target by a slow upward shift of the beam.

The internal beam experiment allows one-due to multiple passing of the beam through the target- to obtain relatively high statistics of the data using a thin target which in turn ensures that the re-scattering and absorption of the reaction products in the target is negligibly small. It also allows one to control the rate of data registration by detectors, which leads to the most efficient performance of the data acquisition system. This was achieved in the present study by controlling the pace of shifting the beam towards the target.

To obtain an absolute normalization of the cross sections the angle and energy integrated differential cross sections $d^{2} \sigma / d \Omega d E$ for ${ }^{7} \mathrm{Be}$ production measured in the present experiment were compared to the total production cross sections known from the compilation of previous data [20]. To ensure the interpolation between individual emission angles as well as extrapolation to the lowest and highest energies of emitted ${ }^{7} \mathrm{Be}$ particles, the present data were parametrized by a simple formula representing the isotropic emission from two sources moving forward along the beam direction (see Appendix of Ref. [1] for details of the parametrization). A very good reproduction of the data by this formula led to a statistical uncertainty of the absolute value of the cross sections not larger than 9\%; however, the uncertainty of the literature value of the production cross section $\sigma\left({ }^{7} \mathrm{Be}\right)$ which is believed to be smaller than $10 \%$ [20], should be also taken into account.

The quality of the absolute normalization was checked by comparison of the total production cross sections from the present experiment at $1.2 \mathrm{GeV}$ proton beam energy with the data measured by Herbach et al. [11], which were obtained by a completely different experimental method, i.e., using an external beam with another detector system and method of absolute normalization. The results obtained in both experiments are shown in Fig. 1. The excellent agreement of the data from both experiments is clearly visible.

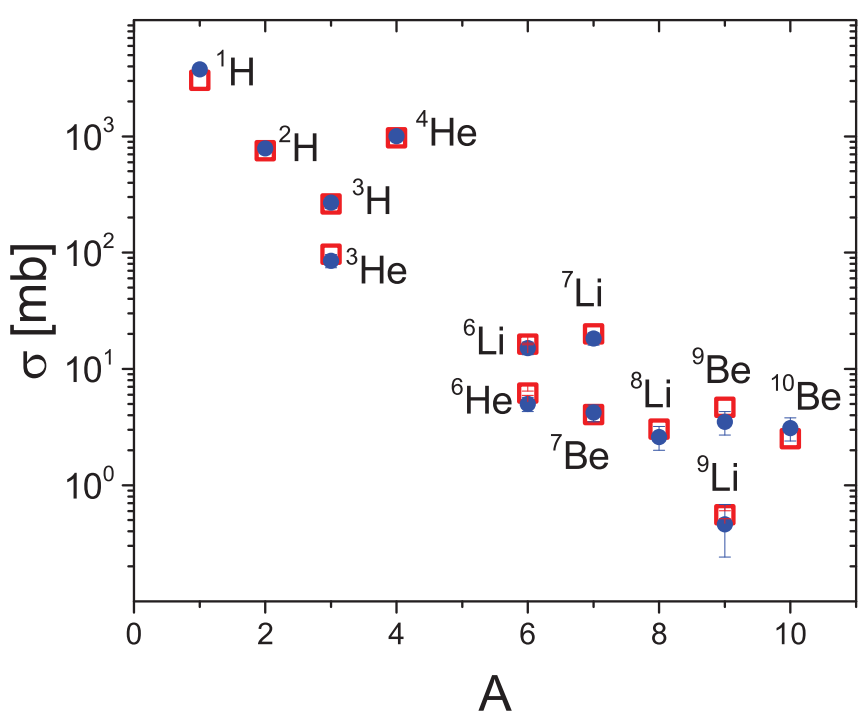

FIG. 1. Comparison of the total cross sections on ${ }^{1-3} \mathrm{H},{ }^{3,4,6} \mathrm{He}$, ${ }^{6-9} \mathrm{Li}$, and ${ }^{7,9,10} \mathrm{Be}$ production from Ref. [11] (blue dots) and those from the present experiment (red squares). Both sets of data were obtained for reactions induced by $1.2 \mathrm{GeV}$ protons on silver target. The double differential cross sections $d^{2} \sigma / d E d \Omega$ were integrated over the full range of angles and over the ejectile kinetic energy range from 0 to $100 \mathrm{MeV}$.

It was possible to compare also the differential cross sections from both experiments, i.e., the data obtained at proton beam energy $1.2 \mathrm{GeV}$ in the present work and those from the measurements of Herbach et al. [11]. However, the data of Ref. [11] were published only in the form of angle integrated energy spectra $d \sigma / d E$ summed over all isotopes of a given element. Therefore, the present data, which were measured as $d^{2} \sigma / d \Omega d E$ for individual isotopes of studied elements, were integrated over angles and summed over isotopes.

The differential data from both experiments are compared in Fig. 2 where cross sections from Ref. [11] are depicted as blue dots and those from the present measurements as red squares. The agreement of all corresponding spectra is excellent. It can be also noticed that significantly better data statistics (smaller statistical uncertainties) was obtained in the present experiment. Because of this it was possible to analyze the present data separately for individual isotopes and in the form of double differential cross sections $d^{2} \sigma / d \Omega d E$.

The double differential cross sections $d^{2} \sigma / d \Omega d E$ measured in the present experiment for a silver target at three proton beam energies $(1.2,1.9$, and $2.5 \mathrm{GeV})$ indicate very similar behavior to that observed in our previous studies for other targets: $\mathrm{Al}[5], \mathrm{Ni}[3,4]$, and $\mathrm{Au}[1,2,4]$. First, they increase systematically with beam energy but the angular dependence of the energy spectra as well as the shape of spectra practically do not change. The relative increase of absolute value of the cross sections is larger for heavier products of the reaction (a factor of 2 to 3 between beam energies 1.2 and $2.5 \mathrm{GeV}$ ) than that for light products (a factor of 1.5 to 2 ). This is illustrated in Fig. 3 for proton, deuteron, and triton spectra measured at $65^{\circ}$ as well as in Fig. 4 for spectra measured at the same angle for isotopes of lithium. Open circles represent the 


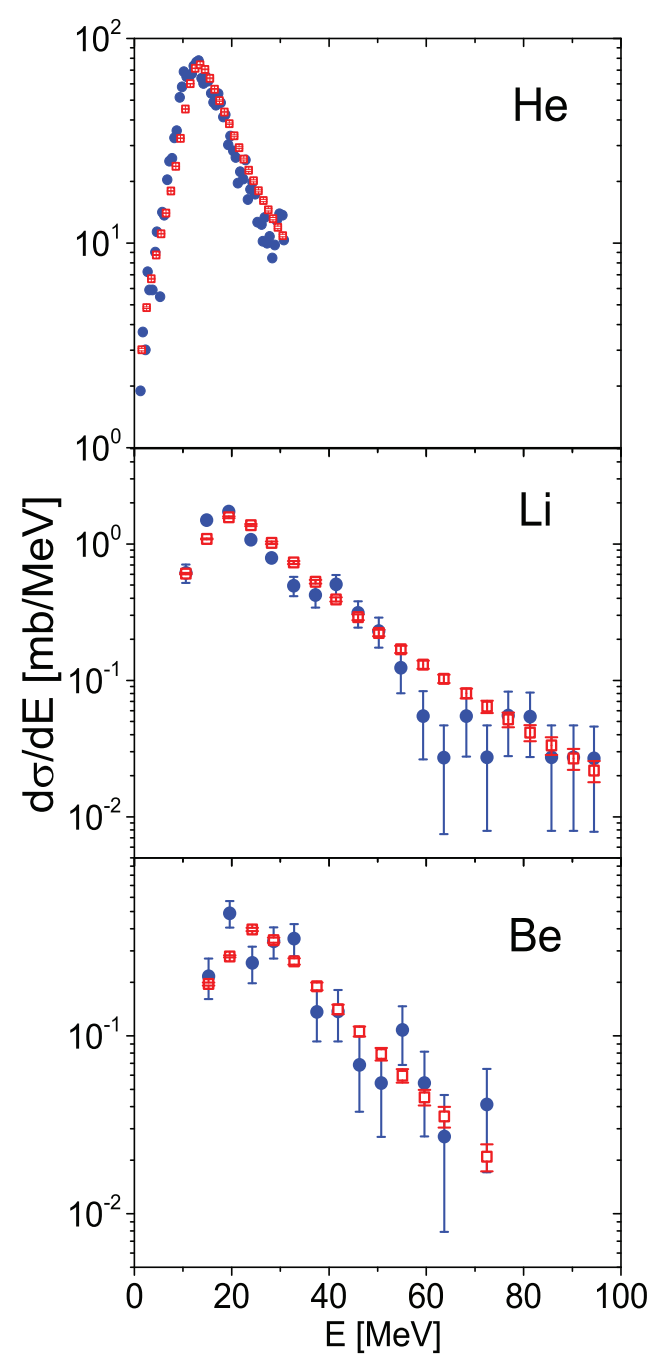

FIG. 2. Comparison of differential cross sections $d \sigma / d E$ summed over all isotopes of $\mathrm{He}$ (top), $\mathrm{Li}$ (middle), and Be (bottom) as obtained in measurements by Herbach et al. [11] (blue dots) and in the present experiment (red squares). The high-energy tail of the He spectrum is not shown because the data from Ref. [11] were cut above $\sim 30 \mathrm{MeV}$.

data obtained at the lowest beam energy $(1.2 \mathrm{GeV})$, the solid circles represent the data at intermediate energy $(1.9 \mathrm{GeV})$, and triangles depict the cross sections measured at $2.5 \mathrm{GeV}$. The energy dependence of data for $\mathrm{He}$ as well as for $\mathrm{Be}, \mathrm{B}$, and heavier IMFs is very similar to that for $\mathrm{H}$ and $\mathrm{Li}$ isotopes shown in Figs. 3 and 4.

The shape of the spectra changes with emission angle: the slope of the spectra increases but their beam energy dependence remains weak for all scattering angles.

Second, it is possible to distinguish two components of the spectra: the low-energy component which is (almost) isotropic and the high-energy one which is forward peaked. This can be observed in Figs. 5 and 6, where the spectra measured at three angles, $20^{\circ}, 65^{\circ}$, and $100^{\circ}$, for $1.9 \mathrm{GeV}$ proton beam energy are depicted for hydrogen and lithium isotopes, respectively.

The proton, deuteron, and triton spectra almost do not change with the emission angle for energies smaller than

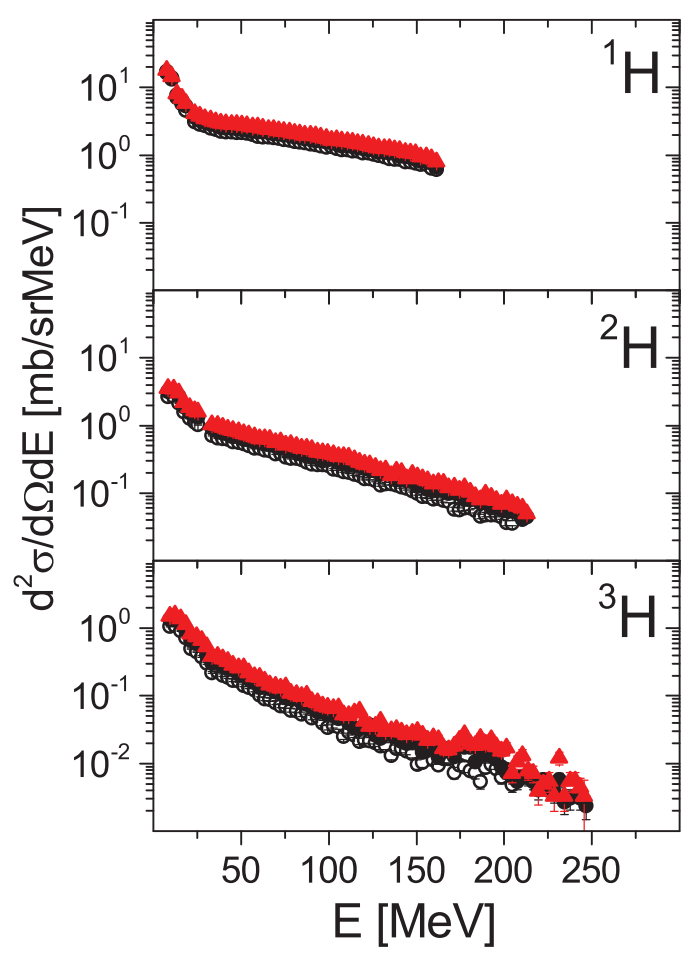

FIG. 3. The beam energy dependence of isotopic hydrogen spectra measured at $65^{\circ}$ for $1.2 \mathrm{GeV}$ (open circles), $1.9 \mathrm{GeV}$ (solid circles), and $2.5 \mathrm{GeV}$ (solid triangles).

25-30 MeV but the higher-energy part decreases clearly with increasing angle. This means that low-energy spectra are almost isotropic, whereas those at higher energy are forward peaked.

Such an effect may be also observed for IMFs but it is less pronounced than for LCPs. It is visible in Fig. 6 where the spectra of lithium isotopes are presented as typical representatives of IMFs.

\section{THEORETICAL ANALYSIS}

The theoretical analysis of the data was performed by assuming a two-step model of the reaction. The first stage of the reaction was treated as an intranuclear cascade of nucleon-nucleon and nucleon-pion collisions which resulted in the emission of nucleons and pions, as well as light charged particles and intermediate mass fragments. The INCL4.6 model [17] was used for this purpose. The emission of particles from equilibrated target remnants of the first stage of the reaction was calculated using four different models: the generalized evaporation model GEM2 of Furihata [21,22], the ABLA07 statistical model of Kelić et al. [23], the statistical multifragmentation model (SMM) of Botvina et al. [24-27], and the sequential binary decay model GEMINI++ of Charity [28-30].

The INCL4.6 model [17] assumes that the nucleon impinging on the target nucleus moves in the square potential well whose radius depends on the nucleon momentum. The depth of the potential well is energy dependent according to the phenomenology of the real part of the nucleon-nucleus opticalmodel potential and is adjusted in such a way as to ensure 


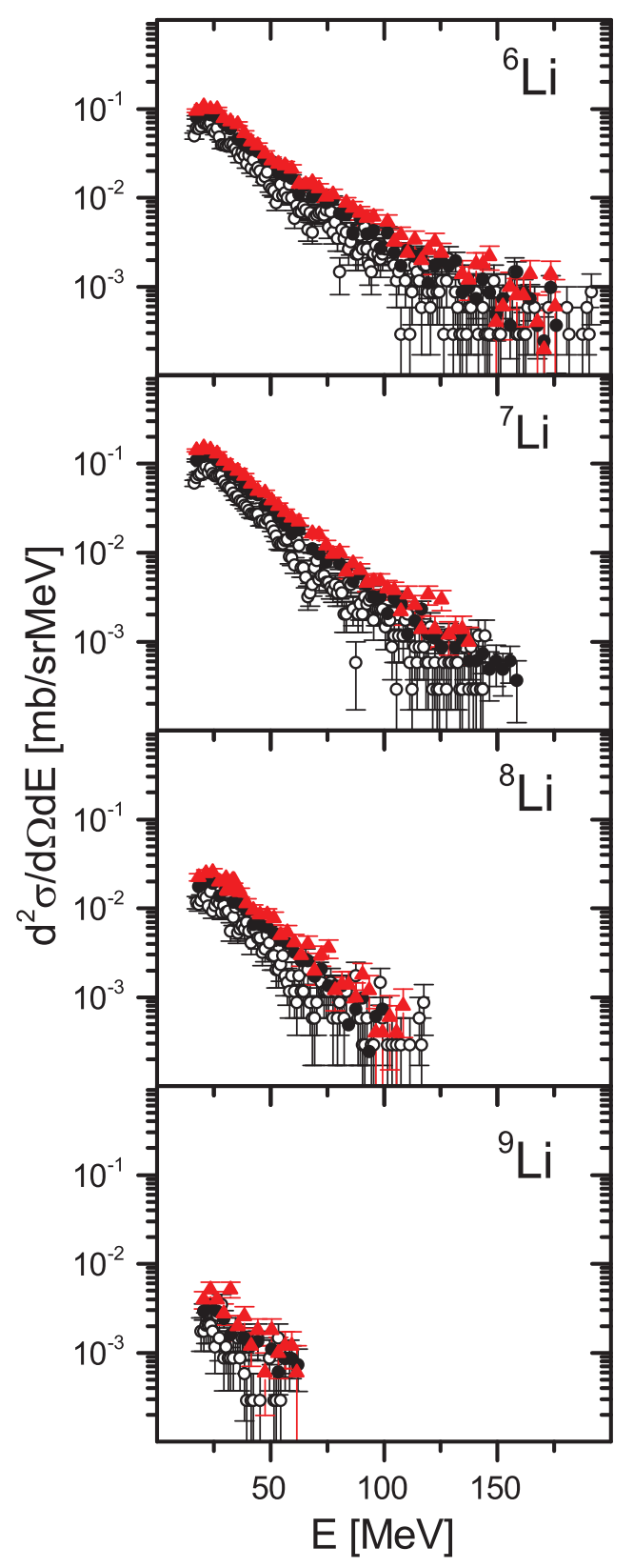

FIG. 4. The beam energy dependence of isotopic lithium spectra measured at $65^{\circ}$ for $1.2 \mathrm{GeV}$ (open circles), $1.9 \mathrm{GeV}$ (solid circles), and $2.5 \mathrm{GeV}$ (solid triangles).

equality of the energy of the neutron and proton Fermi levels. The nucleons move in straight-line trajectories until two of them reach their minimum relative distance, in which case they can scatter with the elastic cross sections of free nucleon-nucleon collisions, or until they reach the surface of the nucleus. In the latter case they can leave the nucleus if their energy is positive and (for protons) if they are able to penetrate the Coulomb barrier. The excitation of the delta resonance in nucleon-nucleon collisions as well as its decay with emission of pions is taken into account. The quantum effects modify this semiclassical description by introducing the Pauli blocking of the collisions which would lead to the occupied states.

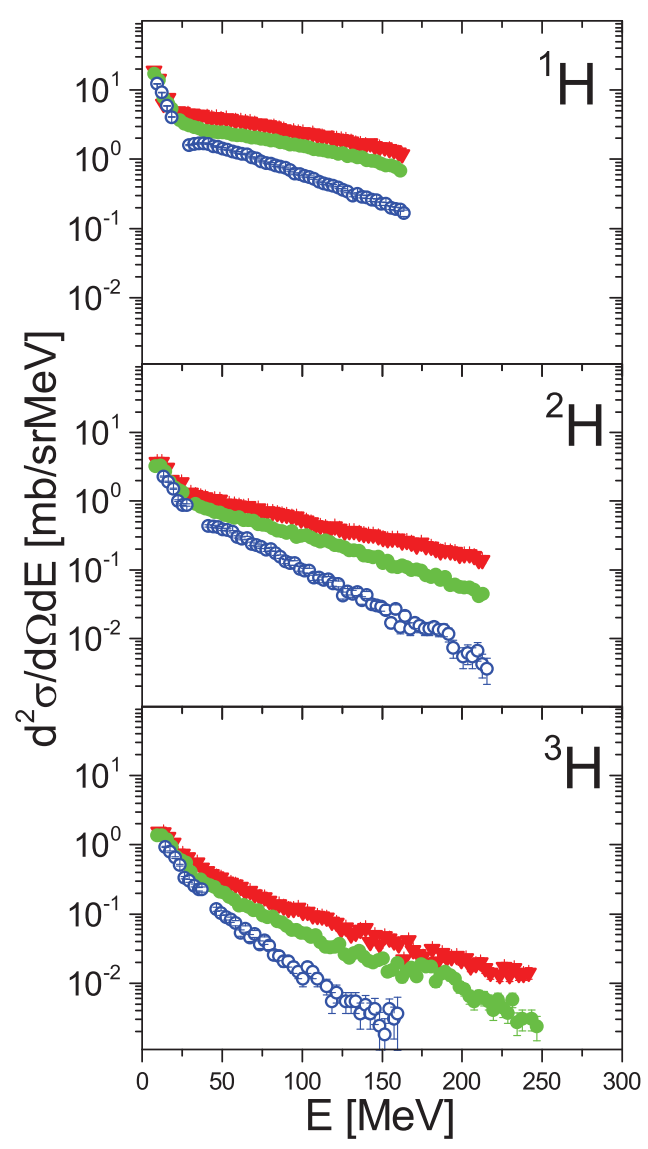

FIG. 5. The angular dependence of isotopic hydrogen spectra measured at proton beam energy $1.9 \mathrm{GeV}$ for $20^{\circ}$ (red triangles), $65^{\circ}$ (solid green circles), and $100^{\circ}$ (open blue circles).

The INCL4.6 model takes into account the possibility to emit particles composed of several nucleons from the stage of the intranuclear cascade. Therefore, it enabled us to calculate at the microscopic level a contribution of preequilibrium processes to the emission of composite particles. This process is treated in the frame of the coalescence model in which nucleons placed at the surface of the nucleus can form, together with the nucleon escaping from the intranuclear cascade, a cluster: composite LCP or light IMF. The clusters with a lifetime longer than $1 \mathrm{~ms}$ are treated as detectable, whereas those with shorter lifetimes are forced to decay. The INCL4.6 model can take into account the emission of IMFs with mass number up to $A=$ 12 ; however, the computation time becomes extremely long for $A>8$ because then the number of possible combinatorial ways to create the emitted particles increases extremely fast. Thus, in the original paper [17] the maximal mass number of emitted clusters is limited by authors of the INCL4.6 program to $A=8$. We followed this prescription and considered in the present work only composite particles with $A<9$. It should be emphasized that such a decision allows us to efficiently perform the calculations; however, it leads to overestimation of the cross sections for the heaviest emitted particles (i.e., those with $A=8$ ) because they effectively contain the contribution from emission of all heavier ejectiles. 


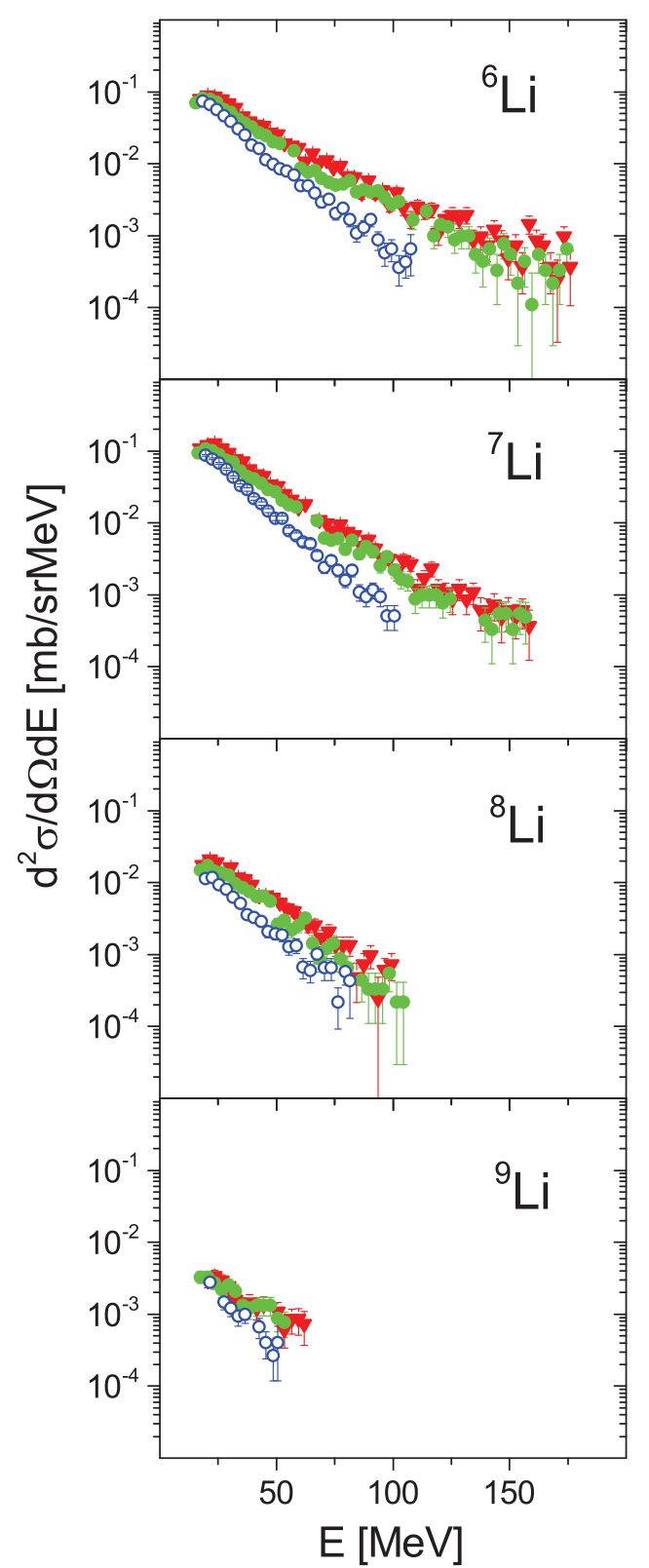

FIG. 6. The angular dependence of isotopic lithium spectra measured at proton beam energy $1.9 \mathrm{GeV}$ for $20^{\circ}$ (red triangles), $65^{\circ}$ (solid green circles), and $100^{\circ}$ (open blue circles).

The four models which were applied for description of the emission of particles from the second step of the reaction, i.e., GEM2, ABLA07, SMM, and GEMINI++, differ in physical assumptions as well as in model realization of the emission scenarios.

The generalized evaporation model GEM2 [21,22] includes sequential evaporation of particles evaluated according to formalism of Weisskopf and Ewing [31] which calculates the probability of particle emission from the energy-dependent cross sections of inverse reactions, level densities of daughter nuclei, as well as Coulomb barriers for charged emitted particles. GEM2 allows also for fission of the excited nucleus in accordance with Atchison's [32] fission model.
The statistical ABLA07 model [23] takes into account the simultaneous breakup of the excited nuclei besides evaporation and fission. Similarly as in GEM2 the evaporation process is calculated according to the Weisskopf-Ewing formalism; however, the ABLA07 model extends it to take into consideration the angular momenta of emitted particles. It is done in an approximate manner by random sampling of the angular momentum change in the evaporation process from a Gaussian distribution with specifically chosen parameters. The fission probability is treated as a diffusion process described by the Fokker-Planck equation. The detailed solution of the equation is analytically parametrized, leading to the timedependent fission width. The multifragmentation mechanism is postulated to appear when the excitation energy per nucleon of the decaying nucleus is larger than $4.2 \mathrm{MeV} /$ nucleon. The process is simulated statistically with thermal expansion of the excited nucleus treated explicitly and sampling of masses and energies of the fragmentation products in line with several phenomenology based prescriptions, e.g., constant $A / Z$ ratio. Details of the computational realization of the model may be found in Ref. [23].

A detailed description of the SMM developed by Botvina et al. [24-26] is provided in Ref. [27]. The model treats the deexcitation of the thermalized remnant of the first step of the proton-nucleus collision as a statistical break-up. The nucleus expands to a certain volume and then breaks up into nucleons and hot fragments. It is assumed that the break-up occurs at a density of one-sixth to one-third of the normal nuclear density. The Monte Carlo method is used for selection of the break-up channel according to probabilities as determined below.

The probability $w_{j}$ of a specific decay channel $j$ of the nucleus excited to the energy $E^{*}$ is proportional to the exponential function of the entropy $S_{j}\left(E^{*}\right)$ :

$$
w_{j} \propto \exp \left(S_{j}\left(E^{*}\right)\right)
$$

Since the model treats the compound nucleus as one of the decay channels the transition from evaporation of particles from the compound nucleus to its multifragmentation is decided on the basis of the available phase space. After break-up of the system, the fragments propagate independently in their mutual Coulomb fields taken into account via the Wigner-Seitz approximation and undergo secondary decays. The deexcitation of large fragments (with mass number larger than 16) is described by the evaporation-fission model, whereas that of smaller fragments is described by the Fermi break-up model [27].

The GEMINI ++ code by Charity [28-30] is a modified version of GEMINI [33] which describes the deexcitation of the compound nucleus by sequential binary decays proceeding until the final products are unable to undergo further division. The Hauser-Feshbach formalism is used to describe an asymmetric decay of the compound nucleus resulting in the emission of light particles (with $Z$ up to 4), whereas Moretto's generalized transition-state formalism [34] is applied to heavier asymmetric divisions. Liquid drop barriers are computed in the frame of the finite-range model [35] with shell and pairing corrections taken from Ref. [36]. The Bohr-Wheeler formalism [37] is used for symmetric fission, and the width of 


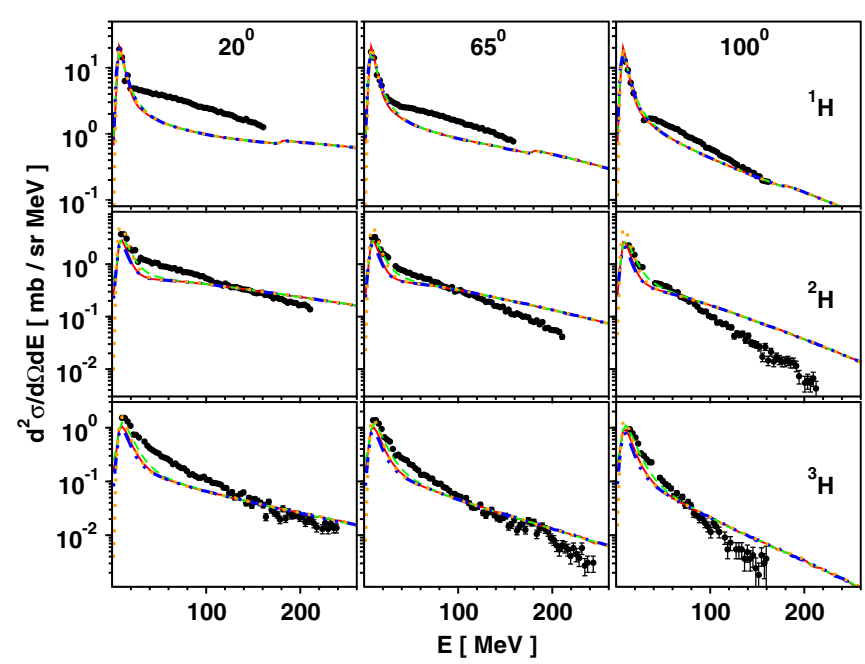

FIG. 7. The solid circles represent experimental isotopic hydrogen spectra measured at proton beam energy $1.9 \mathrm{GeV}$ for $20^{\circ}$ (left), $65^{\circ}$ (middle), and $100^{\circ}$ (right), compared with results of model calculations (lines). The dotted (yellow) line depicts the results of GEM2, the dashed (green) line represents those of ABLA07, the dot-dashed (blue) line represents the results of the GEMINI++ model, and the solid (red) lines correspond to the SMM.

the mass distributions of the fission fragments is interpolated from systematics.

The model calculations are compared in Figs. 7-13 with the data measured at $1.9 \mathrm{GeV}$ proton beam energy. The quality of the description of experimental data is very similar for all beam energies; thus the figures are representative of a full set of the data obtained in the present experiment. The lines shown in the figures depict the sum of the contributions from INCL4.6 and one of the four models of the second stage of the reaction discussed above. The dotted (yellow) line corresponds to GEM2, the dashed (green) line to ABLA07,

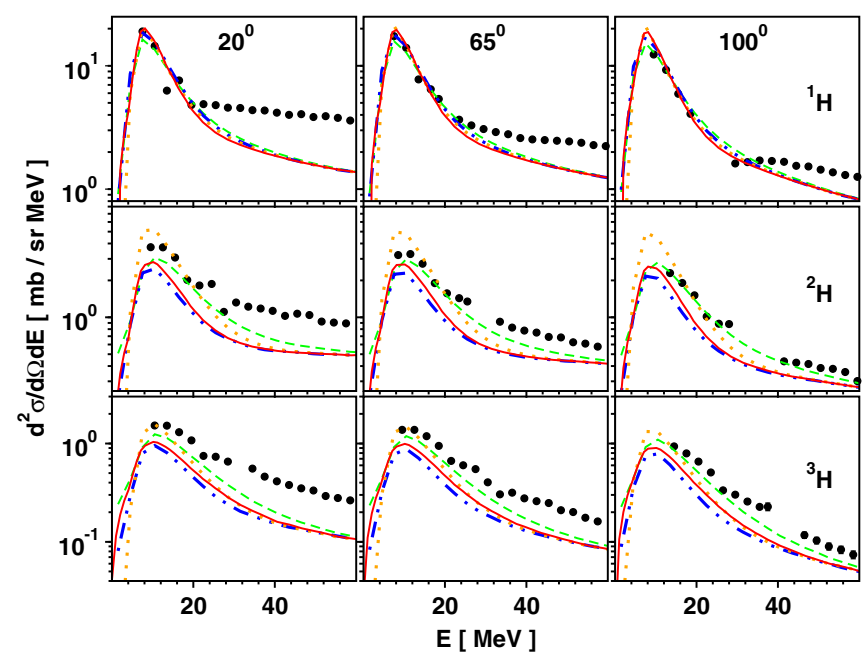

FIG. 8. The same cross sections as in Fig. 7 but presented in the expanded scale of the energy and the cross section to show differences between the data (solid circles) and results obtained with model calculations (lines) for low-energy, isotropic spectra.

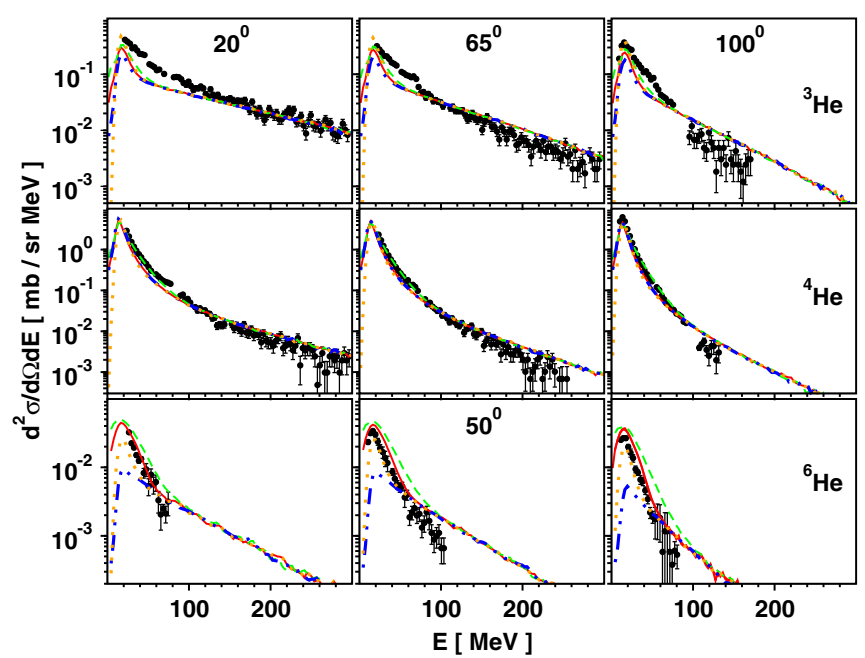

FIG. 9. The same as in Fig. 7 but for helium isotopes. Note that the cross sections of ${ }^{4} \mathrm{He}$ cover almost four orders of magnitude, whereas those of ${ }^{3} \mathrm{He}$ and ${ }^{6} \mathrm{He}$ isotopes vary in the range of two orders of magnitude.

the dash-dotted (blue) line to GEMINI++, whereas the solid (red) line corresponds to the SMM.

It is evident that the high-energy part of all theoretical spectra (energies over $\sim 30 \mathrm{MeV}$ for LCPs and over $\sim 60 \mathrm{MeV}$ for IMFs with mass number A $<9$ ) is dominated by the INCL4.6 contribution because there all lines are indistinguishable. Thus, according to the assumed two-step mechanism of the reaction this range of energies corresponds to preequilibrium processes. The same conclusion may be derived from inspection of the spectra for IMFs with mass number larger than 8 , i.e., the spectra of ${ }^{9} \mathrm{Be},{ }^{10} \mathrm{Be}$, as well as those for all isotopes of $\mathrm{B}$. The experimental cross sections are larger-especially for small scattering angles-than the theoretical ones predicted by models describing the second stage of the reaction.

The theoretical spectra calculated for the small energy region, i.e., energy smaller than $\sim 30 \mathrm{MeV}$ for LCP and smaller than $\sim 60 \mathrm{MeV}$ for IMF, disagree among themselves,

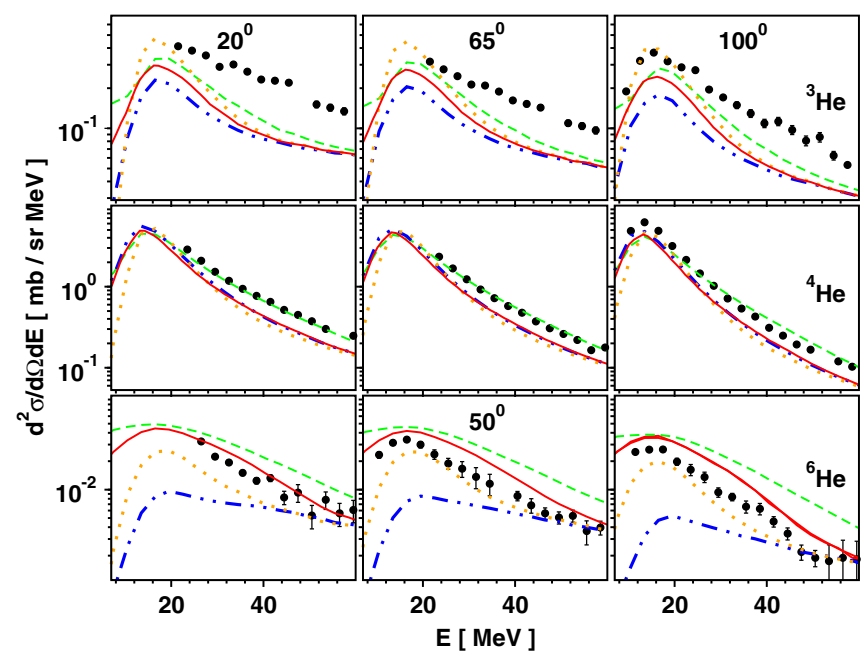

FIG. 10. The same as in Fig. 8 but for helium isotopes. 


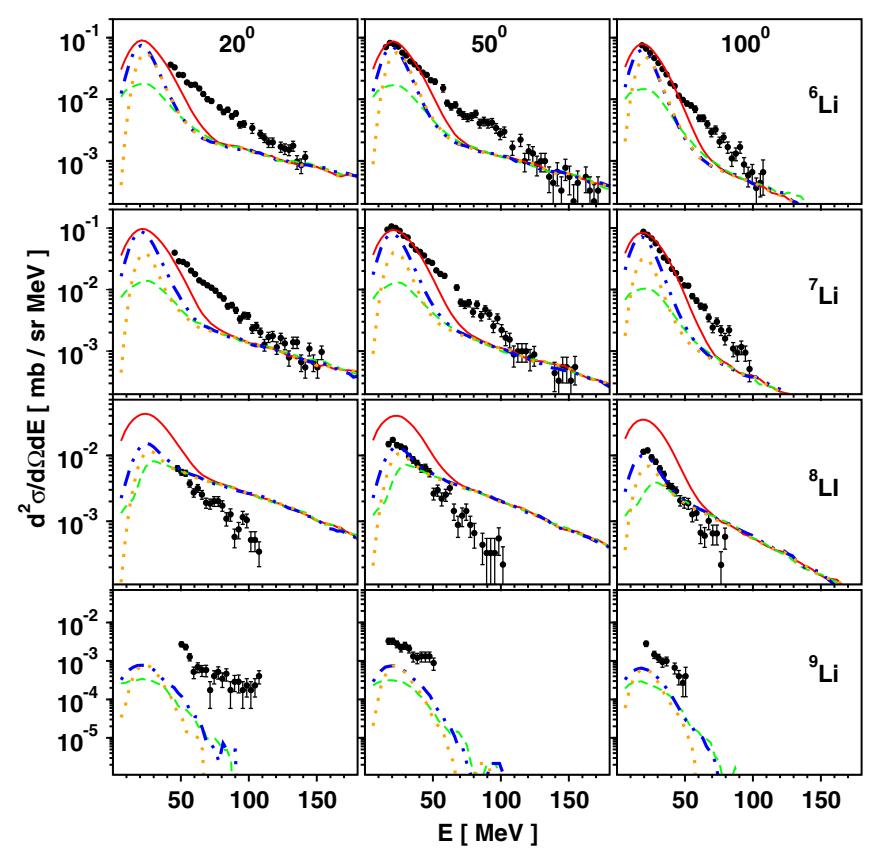

FIG. 11. The solid circles represent experimental isotopic lithium spectra measured at proton beam energy $1.9 \mathrm{GeV}$ for $20^{\circ}$ (left), $50^{\circ}$ (middle), and $100^{\circ}$ (right) compared with results of model calculations (lines). The lines correspond to the same models as in previous figures: the dotted (yellow) line depicts the results of GEM2, the dashed (green) line represents those of ABLA07, the dot-dashed (blue) line corresponds to the GEMINI++ model, and the solid (red) lines correspond to the SMM.

indicating that different models of the deexcitation of the equilibrated remnant of the first stage of the reaction produce different cross sections. This difference increases with the mass number of ejectiles. It is quite significant for all IMFs but is hardly visible in Figs. 7 and 9, which present data for $\mathrm{H}$ and He isotopes, respectively. Therefore, the low-energy part of the spectra for these particles is shown in Figs. 8 and 10 using an expanded scale of the cross sections.

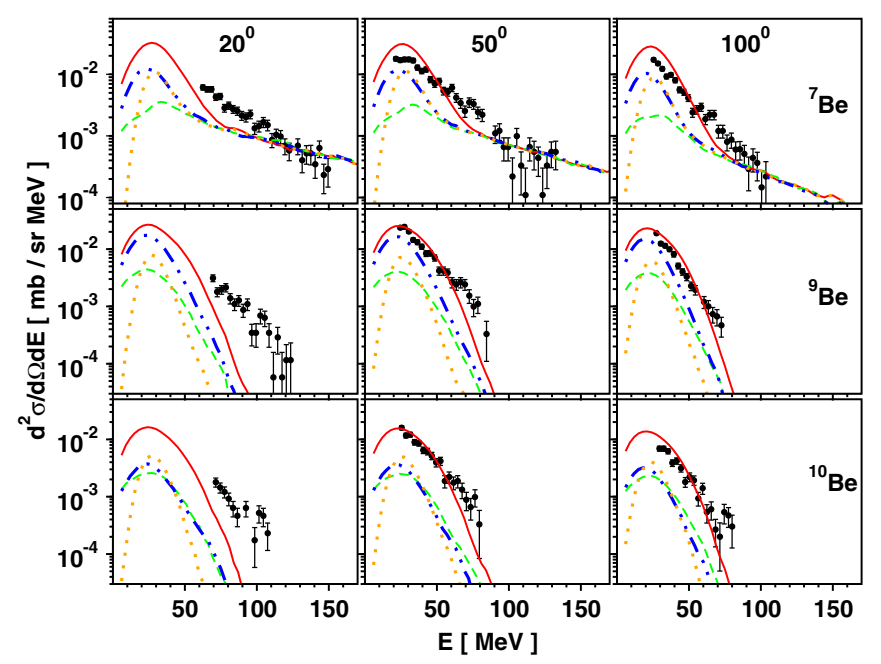

FIG. 12. The same as in Fig. 11 but for beryllium isotopes.

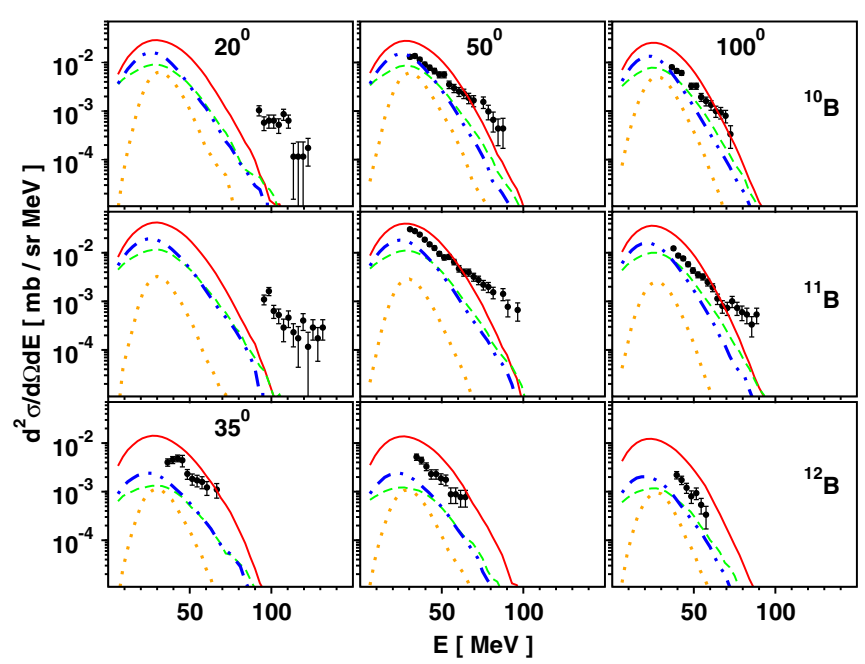

FIG. 13. The same as in Fig. 11 but for boron isotopes. Note that the smallest scattering angle for ${ }^{12} \mathrm{~B}$ is equal to $35^{\circ}$, whereas for ${ }^{10} \mathrm{~B}$ and ${ }^{11} \mathrm{~B}$ it is equal to $20^{\circ}$.

Since the quality of the description of data was found to be different for various elements, the conclusions derived from the analysis are presented separately for each element.

The theoretical spectra for all hydrogen isotopes are less steep than the experimental ones at energies larger than $\sim 30 \mathrm{MeV}$ (cf. Fig. 7). The proton data are there underestimated by the INCL model similarly as it was observed previously for other nuclear systems at GeV energies, e.g., in Refs. [16,17]. The deuteron and triton experimental spectra are underestimated at low but overestimated at high energies. Such an effect is observed at all scattering angles. This may suggest that (i) the coalescence of nucleons into complex particles realized by the INCL4.6 model acts too efficiently, decreasing the yield of protons in favor of the emission of heavier particles, and (ii) the energetic particles are too abundant, decreasing the yield of lower-energy particles.

The low-energy spectra of protons (cf. Fig. 8) are well reproduced by all four models of the second stage of the reaction. Theoretical cross sections are almost exactly the same for all the models, which means that the proton spectra cannot be used for selection of the best model of this stage of the reaction. Another situation is present for deuteron and triton spectra. The sum of INCL4.6 cross sections with those of GEM2 and ABLA07 models is significantly larger than that for the GEMINI++ model and the SMM. The cross sections evaluated with GEM2 are larger than the data and those evaluated with ABLA07 are almost equal to them, whereas the other two models underestimate the data. Since the analysis of the high-energy part of the spectra suggests that the non-equilibrium contribution to the low-energy part of the spectra should be increased, the results obtained with GEMINI++ or the SMM would agree much better with the data than the predictions of GEM2 or ABLA07.

The experimental spectra are qualitatively different for ${ }^{3} \mathrm{He}$, ${ }^{4} \mathrm{He}$, and ${ }^{6} \mathrm{He}$ isotopes. The data for the lightest isotope, i.e., ${ }^{3} \mathrm{He}$, are similar to triton spectra (cf. Figs. 7 and 9). The spectra at low energies are almost angle independent; i.e., 
the equilibrium processes dominate but in the region of higher energies the cross sections decrease with the scattering angle. Such a tendency toward the forward emission of particles is typical for non-equilibrium processes. The same qualitative behavior of spectra is observed for the ${ }^{6} \mathrm{He}$ isotope; however, the non-equilibrium contribution is not as evident as it is for ${ }^{3} \mathrm{He}$. Qualitatively different spectra are present for the ${ }^{4} \mathrm{He}$ particles. The cross sections in the high-energy tail of the spectra are three to four orders of magnitude smaller than in the maxima of the spectra, which means that the equilibrium contribution dominates for this reaction channel. It is worth noticing that the ${ }^{4} \mathrm{He}$ spectra at low energies are well reproduced by all the models of the second stage of the reaction, with ABLA07 slightly superior to the other models (cf. Fig. 10). This is not the case for ${ }^{3} \mathrm{He}$, where all theoretical cross sections are significantly smaller than the data, and for ${ }^{6} \mathrm{He}$, where ABLA07 and SMM overestimate and GEM2 as well as GEMINI++ underestimate the data. It seems that in the last case the non-equilibrium contribution dominates also at the lowest energies and GEMINI++ fails to add an appropriate, significantly large equilibrium cross section. The disagreement of the INCL+GEMINI calculations with the ${ }^{6} \mathrm{He}$ data was also observed previously, e.g., in the papers of Herbach et al. [11] and Fidelus et al. [5].

The experimental spectra of $\mathrm{Li}$ isotopes $\left({ }^{6} \mathrm{Li},{ }^{7} \mathrm{Li},{ }^{8} \mathrm{Li}\right.$, and ${ }^{9} \mathrm{Li}$ ) are presented in Fig. 11 (solid circles) together with theoretical spectra (lines). In this figure as well as in Figs. 12 and 13 the data at $50^{\circ}$ are shown instead of those at $65^{\circ}$ as was done for $\mathrm{H}$ and $\mathrm{He}$ data in the previous figures.

The theoretical cross sections of ${ }^{8} \mathrm{Li}$ are artificially increased by the fact that the present INCL4.6 calculations attribute non-equilibrium cross sections of all ejectiles with mass number $A \geqslant 8$ to the channel with $A=8$. The shape of non-equilibrium spectra of ${ }^{8} \mathrm{Li}$ is practically the same as that for ${ }^{6} \mathrm{Li}$ and ${ }^{7} \mathrm{Li}$; thus it may be conjectured that the shape of ${ }^{8} \mathrm{Li}$ spectra is not deformed by the above deficiency of the calculations. It is evident that the slope of all non-equilibrium spectra is too small in comparison to the experimental ones. As a result the theory underestimates significantly the data of ${ }^{6} \mathrm{Li}$ and ${ }^{7} \mathrm{Li}$ in the energy range from $\sim 60$ to $\sim 120 \mathrm{MeV}$. Such a conclusion is probably true also for ${ }^{8} \mathrm{Li}$ but the uncertainty of the magnitude of the theoretical cross section for $A=8$ (see comment above) prohibits us from deriving such a statement. The equilibrium contribution to the spectra is localized at low energies (smaller than $\sim 60 \mathrm{MeV}$ ) and has a similar bell-like shape for all models, with the exception of the SMM, which produces slightly broader spectra. The magnitude of this contribution is the largest for the SMM, slightly smaller for GEMINI++, and significantly smaller for GEM2 and ABLA07 in the quoted sequence. It is worth noticing that the SMM predicts negligibly small equilibrium cross sections for ${ }^{9} \mathrm{Li}$, which does not agree with the data.

The experimental spectra of ${ }^{7} \mathrm{Be}$ have the same shape and angular dependence as the data for ${ }^{7} \mathrm{Li}$ with the absolute value of the cross sections approximately five times smaller. The same differences between model ${ }^{7} \mathrm{Be}$ cross sections and the data are observed as those for ${ }^{7} \mathrm{Li}$. The ${ }^{9} \mathrm{Be}$ and ${ }^{10} \mathrm{Be}$ data could be compared only with equilibrium theoretical cross sections because the calculations of the non-equilibrium contribution with INCL4.6 was limited to ejectiles with mass number $A \leqslant 8$. It is obvious from the inspection of Fig. 12 that the theoretical cross sections underestimate the ${ }^{9} \mathrm{Be}$ and ${ }^{10} \mathrm{Be}$ data at $20^{\circ}$ as well as at $50^{\circ}$. This effect is very strong at the smaller of these two angles and still significant at the larger of them. Therefore, it may be stated that the non-equilibrium processes give quite a significant contribution to the reaction. The SMM calculations provide the largest cross sections of equilibrium processes for all Be isotopes. The GEMINI++, GEM2, and ABLA07 results are two to three times smaller than those of SMM; however, the relationships among cross sections of these three models vary for different $\mathrm{Be}$ isotopes. It is impossible to claim which of them is most reliable because the unknown non-equilibrium contribution may strongly influence the conclusions.

The experimental spectra of ${ }^{10} \mathrm{~B},{ }^{11} \mathrm{~B}$, and ${ }^{12} \mathrm{~B}$ (dots) are shown in Fig. 13 together with theoretical spectra (lines) calculated in the frame of four models of the second stage of the reaction. The relative magnitude of the individual models there is the same as for Be isotopes; i.e., the SMM predicts the largest cross sections-larger by a factor 2 to 3 than other models for ${ }^{10} \mathrm{~B}$ and ${ }^{11} \mathrm{~B}$ - whereas this difference is still larger (even one order of magnitude) for ${ }^{12} \mathrm{~B}$. Results of GEMINI++ and ABLA07 are very similar, whereas those of GEM2 are the smallest and cover a smaller energy range than the other models. The data for $20^{\circ}$ are strongly underestimated by all the models, by more than one order of magnitude. Such an underestimation is also visible for larger scattering angles; however, it is strong only for GEMINI++, GEM2, and ABLA07, whereas predictions of the SMM underestimate the data at large energies (larger than $\sim 80 \mathrm{MeV}$ for ${ }^{10} \mathrm{~B}$ and ${ }^{11} \mathrm{~B}$ ) but are larger than data at smaller energies for all $\mathrm{B}$ isotopes. It indicates that SMM cross sections are too large because the contribution of non-equilibrium processes is expected to add incoherently to that of the equilibrium one.

The lack of the theoretical non-equilibrium contribution in the analysis of $\mathrm{Be}$ (isotopes with $A=9$ and 10) and $\mathrm{B}$ spectra should not influence the present conclusions since the shape of theoretical non-equilibrium spectra as well as their angular dependence does not change significantly with the mass number of ejectiles. Thus, it seems reasonable to conjecture that the theoretical non-equilibrium contribution for these isotopes is small below the evaporation peak as is the case for lighter isotopes.

The qualitative comparison of the data and model cross sections should be accompanied by some quantitative arguments. This is especially important for the situation when only part of the data and calculations is presented in the figures, namely, that corresponding to the proton beam energy of $1.9 \mathrm{GeV}$. The $A$ deviation factor proposed in Ref. [19] was used for the quantitative comparison of the data and model cross sections. This factor is defined by the following formula:

$$
A \equiv \frac{1}{N} \sum_{i=1}^{N} \frac{\left|\sigma_{i}^{\text {expt }}-\sigma_{i}^{\mathrm{cal}}\right|}{\sigma_{i}^{\mathrm{expt}}+\sigma_{i}^{\mathrm{cal}}}
$$

where $\sigma_{i}^{\text {expt }}$ and $\sigma_{i}^{\text {cal }}$ denote the experimental and theoretical differential cross sections $d^{2} \sigma / d \Omega d E$, respectively. The index $i$ denotes all scattering angles and energy bins for a given ejectile. 

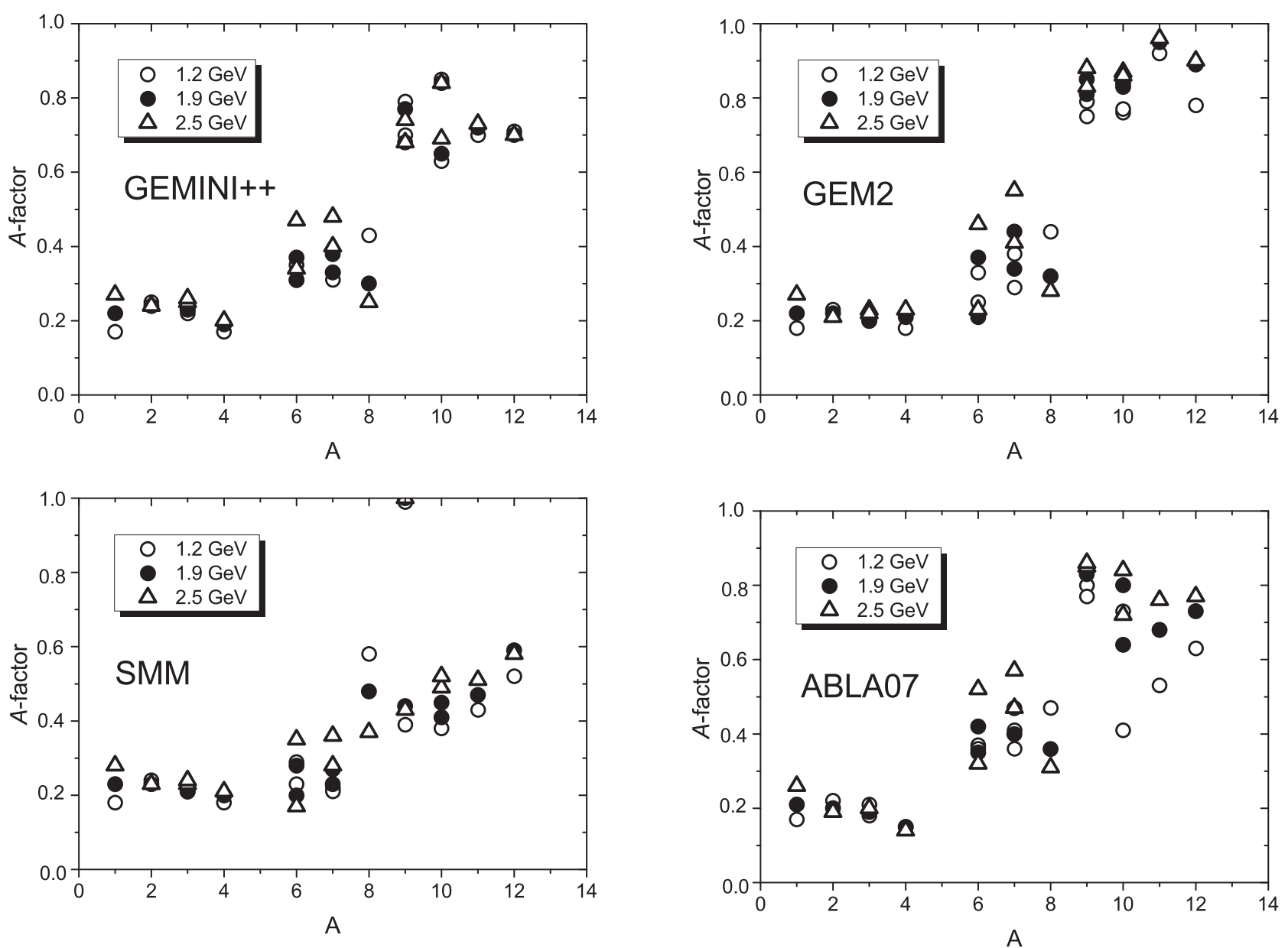

FIG. 14. The deviation $A$ factor as a function of the mass number of ejectiles. Results obtained for different models are presented in separate panels: GEMINI++ in the top left, followed by GEM2, ABLA07, and SMM in clockwise order, respectively. The data and calculations for $1.2 \mathrm{GeV}$ proton beam energy are denoted by open circles, those for $1.9 \mathrm{GeV}$ by solid circles, and those for $2.5 \mathrm{GeV}$ by open squares.

The $A$ factor has the following attractive properties:

(i) Its value is independent of the absolute value of the cross sections, which is important in the case where the cross sections cover a large range of values.

(ii) It is not influenced by the fact that some of the data are overestimated by the model cross sections and other data are underestimated.

(iii) Its value belongs to the interval $[0,1]$ being equal to zero when all calculated cross sections are equal to corresponding experimental cross sections and increases with increase of the differences between the data and theoretical values. Very small values of the $A$ factor correspond to the situation when $\sigma_{i}^{\text {cal }} \approx \sigma_{i}^{\text {expt }}$; therefore, the $A$-factor value may be then interpreted as half of the average relative distance between the experimental and theoretical cross sections.

The $A$-factor values calculated for all the data from the present experiment are collected in Fig. 14 and depicted as a function of the mass number of ejectiles. The results obtained using INCL4.6 coupled to GEMINI++ are presented in the top left panel of the figure whereas those obtained with
GEM2, ABLA07, and SMM are shown in the top right, bottom right, and bottom left panels, respectively. The open circles correspond to the data measured at $1.2 \mathrm{GeV}$ proton beam energy, the solid circles to the data at $1.9 \mathrm{GeV}$, and the open squares to the data at $2.5 \mathrm{GeV}$.

It is possible to derive several interesting conclusions from the inspection of Fig. 14:

(i) The quality of agreement between the data and model calculations is practically the same for all proton beam energies.

(ii) The $A$-factor values are similar for all theoretical models; thus none of them may be favored. The exception is only visible for particles with the mass number $A=10-12$, where the $A$-factor values are smaller for the SMM than for the other models. This effect should be, however, attributed not to the good reproduction of the data ( $A$ factor is large) but to the fact that the theoretical cross sections of the SMM overestimate on average these data whereas other models underestimate them. Then for the same set of data and a similar deviation between the data and model cross sections, the denominator in the definition 
of the $A$ factor [cf. Eq. (1)] is larger for the SMM than for other models, thus leading to the smaller value of this factor.

(iii) None of the models may be validated since minimal values of the $A$ factor are equal to about 0.2 , which means that the average relative distance between the data and the theoretical cross sections is larger than $\sim 40 \%$.

(iv) Three groups of ejectiles which differ significantly by value of the $A$ factor are visible in the figure: the LCP with $A$ factor $\approx 0.2$, the IMF with mass number $A$ not larger than 8 for which the $A$ factor $\approx 0.4$, and the IMF with mass numbers $A>8$ for which the $A$ factor $\approx 0.5-0.8$. There is a simple and natural explanation of the presence of such distinct groups of ejectiles. The coalescence mechanism reproduces reasonably well the non-equilibrium part of the LCP spectra, whereas this is not true for IMFs with mass number $A \leqslant 8$. Furthermore, the data with mass number $A>8$ are compared only with the equilibrium cross sections. The increase of the $A$-factor value for these particles indicates the presence of significant (neglected in the present analysis) contribution of a non-equilibrium mechanism to the data.

\section{SUMMARY AND CONCLUSIONS}

The double differential cross sections $d^{2} \sigma / d \Omega d E$ for light charged particles and intermediate mass fragments produced in $p+\mathrm{Ag}$ collisions were measured at three proton energies: $T_{p}=1.2,1.9$, and $2.5 \mathrm{GeV}$. The spectra of protons, deuterons, tritons, ${ }^{3,4,6} \mathrm{He},{ }^{6-9} \mathrm{Li},{ }^{7,9,10} \mathrm{Be}$, and ${ }^{10-12} \mathrm{~B}$ were determined at seven scattering angles from $15.6^{\circ}$ to $100^{\circ}$. To our knowledge the present results form the only such set of the double differential cross sections $d^{2} \sigma / d \Omega d E$ measured for an $\mathrm{Ag}$ target in the $1.2-2.5 \mathrm{GeV}$ energy range besides the data of Herbach et al. [11], which were determined at $1.2 \mathrm{GeV}$ with significantly smaller statistics. Our data measured at this energy agree perfectly with the data of Herbach et al.

The shape of the experimental spectra as well as their angular dependence vary smoothly with the beam energy in similar way to our previous results obtained at this beam energy range for heavier $(\mathrm{Au}[1,2])$ and lighter $(\mathrm{Ni}[3,4]$ as well as Al [5]) targets. The low-energy part of the energy spectra of all ejectiles is almost angle independent, whereas the high-energy part is anisotropic: the forward direction of emission of the high-energy particles is clearly favored. This qualitative property of the data indicates the presence of a significant contribution of non-equilibrium processes to the reaction mechanism. The energy dependence of the absolute value of the cross sections observed in the present study is weaker than for $\mathrm{Au}$ but stronger than for $\mathrm{Ni}$ and $\mathrm{Al}$. It is in line with the statement that the leveling of the total production cross sections appears at lower beam energies for light targets than for heavy ones (cf., for example, Ref. [20]).

The present data were compared to predictions of the microscopic, two-step model of the reaction using default parameters for both stages of the process. The first, nonequilibrium step of the process was described by the INCL4.6 model [17], which takes into consideration the intranuclear cascade of nucleon-nucleon and nucleon-pion collisions as well as the emission of complex particles created due to the coalescence of the nucleon escaping from the cascade with other nucleons which are sufficiently close to it in the coordinate and momentum space. The deexcitation of the equilibrated remnant of the first stage of the reaction was calculated by means of four different models, namely, GEM2 [21,22], ABLA07, [23], GEMINI++ [28-30], and SMM [24-27].

It was found from the quantitative analysis using the $A$ deviation factor [cf. Eq. (1)] that the agreement between the data and model cross sections is not satisfactory, especially for intermediate mass fragments (cf. Fig. 14); thus the applied theoretical models cannot be validated.

The inspection of Figs. 7-13 indicated that such a conclusion is mainly due to a disagreement of the data and model cross sections for forward scattering angles and the high-energy part of the spectra. The low-energy, isotropic part of the spectra ( $E<30 \mathrm{MeV}$ for LCPs as well as $E<50 \mathrm{MeV}$ for IMFs) could be qualitatively reproduced by the mechanism which assumed emission of particles from equilibrated, excited remnants of the first, non-equilibrium stage of the reaction. However, the high-energy tails of all theoretical spectra were too flat in comparison to the experimental ones, which led to the underestimation of the data at the energy range from $\sim 30-60 \mathrm{MeV}$ to $\sim 150 \mathrm{MeV}$ and overestimation of the data at higher energies. This common property of all the spectra shows that another mechanism of the non-equilibrium processes has to be proposed to achieve satisfactory agreement with the data. Since similar effects were observed for proton induced reactions in the proton beam energy range $1.2-2.5 \mathrm{GeV}$ for heavier than the Ag targets (Au [1,2]) as well as for the lighter ones ( $\mathrm{Ni}[3,4], \mathrm{Al}[5])$ it seems that the non-equilibrium processes contribute significantly to these reactions for all target nuclei. Moreover, it may be conjectured that they are present also at other proton beam energies. For example, the above discussed effects are very similar to those found in the recent analogous analysis of the intermediate mass fragments spectra and angular distributions from $p+\mathrm{Ag}$ collisions at $480 \mathrm{MeV}$ proton beam energy [18] as well as isotopically identified production cross sections of heavy products from ${ }^{136} \mathrm{Xe}+\mathrm{H}$ reactions at $500 \mathrm{MeV} /$ nucleon energy of ${ }^{136} \mathrm{Xe}$ [19]. Thus the explanation of the nature of the non-equilibrium processes in proton induced reactions at $\mathrm{GeV}$ energies remains an important and intriguing problem to be solved.

The numerically tabulated histograms of the present data are available upon request.

\section{ACKNOWLEDGMENTS}

The technical support of A. Heczko, W. Migdał, and $\mathrm{N}$. Paul in preparation of experimental apparatus is greatly appreciated. This work was supported by the European Commission through the European Community-Research Infrastructure Activity under FP6 project Hadron Physics (Contract No. RII3-CT-2004-506078), and HadronPhysics2 (Contract No. 227431). We thank the code developers and authors A. Boudard, J. Cugnon, J. C. David, S. Leray, and D. Mancusi for 
providing us with the latest version of their Liege intranuclear cascade code INCL4.6. We are also grateful to A. Kelić-Heil for the improved version of ABLA07. One of us (S.K.S.) acknowledges gratefully the support by the Foundation for Polish Science-MPD program, co-financed by the European Union within the European Regional Development Fund.
[1] A. Bubak, A. Budzanowski, D. Filges, F. Goldenbaum, A. Heczko, H. Hodde, L. Jarczyk, B. Kamys, M. Kistryn, St. Kistryn, St. Kliczewski, A. Kowalczyk, E. Kozik, P. Kulessa, H. Machner, A. Magiera, W. Migdał, N. Paul, B. Piskor-Ignatowicz, M. Puchała, K. Pysz, Z. Rudy, R. Siudak, M. Wojciechowski, and P. Wüstner, Phys. Rev. C 76, 014618 (2007).

[2] A. Budzanowski, M. Fidelus, D. Filges, F. Goldenbaum, H. Hodde, L. Jarczyk, B. Kamys, M. Kistryn, St. Kistryn, St. Kliczewski, A. Kowalczyk, E. Kozik, P. Kulessa, H. Machner, A. Magiera, B. Piskor-Ignatowicz, K. Pysz, Z. Rudy, R. Siudak, and M. Wojciechowski, Phys. Rev. C 78, 024603 (2008).

[3] A. Budzanowski, M. Fidelus, D. Filges, F. Goldenbaum, H. Hodde, L. Jarczyk, B. Kamys, M. Kistryn, St. Kistryn, St. Kliczewski, A. Kowalczyk, E. Kozik, P. Kulessa, H. Machner, A. Magiera, B. Piskor-Ignatowicz, K. Pysz, Z. Rudy, R. Siudak, and M. Wojciechowski, Phys. Rev. C 80, 054604 (2009).

[4] A. Budzanowski, M. Fidelus, D. Filges, F. Goldenbaum, H. Hodde, L. Jarczyk, B. Kamys, M. Kistryn, St. Kistryn, St. Kliczewski, A. Kowalczyk, E. Kozik, P. Kulessa, H. Machner, A. Magiera, B. Piskor-Ignatowicz, K. Pysz, Z. Rudy, R. Siudak, and M. Wojciechowski, Phys. Rev. C 82, 034605 (2010).

[5] M. Fidelus, D. Filges, F. Goldenbaum, H. Hodde, A. Jany, L. Jarczyk, B. Kamys, M. Kistryn, St. Kistryn, St. Kliczewski, E. Kozik, P. Kulessa, H. Machner, A. Magiera, B. PiskorIgnatowicz, K. Pysz, Z. Rudy, Sushil K. Sharma, R. Siudak, and M. Wojciechowski, Phys. Rev. C 89, 054617 (2014).

[6] R. E. L. Green and R. G. Korteling, Phys. Rev. C 18, 311 (1978).

[7] R. E. L. Green and R. G. Korteling, Phys. Rev. C 22, 1594 (1980).

[8] R. E. L. Green, K. P. Jackson, and R. G. Korteling, Phys. Rev. C 25, 828 (1982).

[9] R. E. L. Green, R. G. Korteling, and K. P. Jackson, Phys. Rev. C 29, 1806 (1984).

[10] R. E. L. Green, R. G. Korteling, J. M. D‘Auria, K. P. Jackson, and R. L. Helmer, Phys. Rev. C 35, 1341 (1987).

[11] C.-M. Herbach, D. Hilscher, U. Jahnke, V. G. Tishchenko, J. Galin, A. Letourneau, A. Péghaire, D. Filges, F. Goldenbaum, L. Pieńkowski, W. U. Schröder, and J. Töke, Nucl. Phys. A 765, 426 (2006).

[12] E. K. Hyde, G. W. Butler, and A. M. Poskanzer, Phys. Rev. C 4, 1759 (1971).

[13] A. Letourneau, A. Böhm, J. Galin, B. Lott, A. Péghaire, M. Enke, C.-M. Herbach, D. Hilscher, U. Jahnke, V. Tishchenko et al., Nucl. Phys. A 712, 133 (2002).

[14] G. D. Westfall, R. G. Sextro, A. M. Poskanzer, A. M. Zebelman, G. W. Butler, and E. K. Hyde, Phys. Rev. C 17, 1368 (1978).
[15] A. M. Poskanzer, G. W. Butler, and E. K. Hyde, Phys. Rev. C 3, 882 (1971)

[16] A. Boudard, J. Cugnon, S. Leray, and C. Volant, Nucl. Phys. A 740, 195 (2004).

[17] A. Boudard, J. Cugnon, J. C. David, S. Leray, and D. Mancusi, Phys. Rev. C 87, 014606 (2013).

[18] S. K. Sharma, B. Kamys, F. Goldenbaum, and D. Filges, Eur. Phys. J. A 52, 171 (2016).

[19] S. K. Sharma, B. Kamys, F. Goldenbaum, and D. Filges, Eur. Phys. J. A 53, 150 (2017)

[20] A. Bubak, B. Kamys, M. Kistryn, and B. Piskor-Ignatowicz, Nucl. Instrum. Methods Phys. Res., Sect. B 226, 507 (2004).

[21] S. Furihata, Nucl. Instrum. Methods Phys. Res., Sect. B 171, 251 (2000).

[22] S. Furihata and T. Nakamura, J. Nucl. Sci. Technol. Suppl. 39, 758 (2002).

[23] A. Kelić, M. V. Ricciardi, and K.-H. Schmid, Proceedings of the Joint ICTP-IAEA Advanced Workshop on Model Codes for Spallation Reactions, ICTP Trieste, Italy, 4-8 February 2008, edited by D. Filges et al., (IAEA, Vienna, 2008), p. 181.

[24] A. S. Botvina, A. S. Iljinov, and I. N. Mishustin, Yad. Fiz. 42, 1127 (1985) [Sov. J. Nucl. Phys. 42, 712 (1985)].

[25] A. S. Botvina, A. S. Iljinov, I. N. Mishustin, J. P. Bondorf, R. Donangelo, and K. Sneppen, Nucl. Phys. A 475, 663 (1987).

[26] A. S. Botvina, A. S. Iljinov, and I. N. Mishustin, Nucl. Phys. A 507, 649 (1990).

[27] J. P. Bondorf, A. S. Botvina, A. S. Iljinov, I. N. Mishustin, and K. Sneppen, Phys. Rep. 257, 133 (1995).

[28] R. J. Charity, Proceedings of the Joint ICTP-IAEA Advanced Workshop on Model Codes for Spallation Reactions, ICTP Trieste, Italy, 4-8 February 2008, edited by D. Filges et al. (IAEA, Vienna, 2008), p. 139.

[29] R. J. Charity, Phys. Rev. C 82, 014610 (2010).

[30] D. Mancusi, R. J. Charity, and J. Cugnon, Phys. Rev. C 82, 044610 (2010).

[31] V. F. Weisskopf and D. H. Ewing, Phys. Rev. 57, 472 (1940; 57, $935(1940$

[32] F. Atchison, Proceedings of the Meeting on Targets for Neutron Beam Spallation Source, edited by G. Bauer (KFA Jülich Germany, Jül-conf-34, 1980), p. 17.

[33] R. Charity, M. A. McMahan, G. J. Wozniak, R. J. McDonald, L. G. Moretto, D. G. Sarantites, L. G. Sobotka, G. Guarino, A. Pantaleo, L. Fiore et al., Nucl. Phys. A 483, 371 (1988).

[34] L. G. Moretto, Nucl. Phys. A 247, 221 (1975); L. G. Moretto and G. J. Wozniak, Prog. Part. Nucl. Phys. 21, 401 (1988).

[35] A. J. Sierk, Phys. Rev. C 33, 2039 (1986).

[36] P. Möller et al., At. Data Nucl. Data Tables 59, 185 (1995).

[37] N. Bohr and J. A. Wheeler, Phys. Rev. 56, 426 (1939). 